\title{
Assessment of Taste Sensation After Tonsillectomy Operation
}

\author{
Abd El-Hay Rashad AlAsy ${ }^{1}$, Ayman El-Said Abd Elaziz ${ }^{1}$, Heba Abd EIrheem AboEl-Naga ${ }^{1}$, \\ Mohammed Mostafa Abed ${ }^{2,}$ \\ ${ }^{1}$ Department of Otolaryngology, Faculty of Medicine, Menoufia University, Menoufia, Egypt \\ ${ }^{2}$ Department of Otolaryngology, Shebin Elkom Teaching Hospital, Menoufia, Egypt
}

Email address:

mohamed.3abed@gmail.com (M. M. Abed),Hebarehem@yahoo.com (H. A. A.-Naga)

\section{To cite this article:}

Abd El-Hay Rashad AlAsy, Ayman El-Said Abd Elaziz, Heba Abd Elrheem AboEl-Naga, Mohammed Mostafa Abed. Assessment of Taste Sensation After Tonsillectomy Operation. American Journal of Clinical and Experimental Medicine. Vol. 3, No. 5, 2015, pp. 241-246. doi: 10.11648/j.ajcem.20150305.18

\begin{abstract}
Objectives: The chemical senses and their normal functioning constitute a necessity for a good quality of life as the gustatory system influence to a large extent food selection and affect nutrition. Altered taste perception seems to be more frequent than previously thought. Fortunately, most of prospective studies show that it remains a transient phenomenon with total recovery after several weeks. Background: It is well known that tonsillectomy is the most commonly performed operation in the otolaryngologicalfield. Although tonsillectomy is regarded as a safe surgery, it still has the risk of per- and postoperative complications. However, taste disturbance is an unusual complication with few reports on it. Patients and methods: This study was carried on 100 patients ( 60 females and 40 males).planned to do bilateral tonsillectomy, they was between 10 to 49 years of age. Chemogustometry was done one day prior to operation to assure intact and efficient taste sensation. Then one, seven, fifteen and thirteen days respectively post operative as follow up in abnormal findings. Four tastants with two concentrations for each were used: quininsulfate or caffeine $(0.075 \% ; 0.2 \%)=>$ for bitter taste, glucose $(2 \% ; 10 \%)=>$ for sweet taste, citric acid $(0.5 \% ; 7.5 \%)=>$ for sour taste, sodium chloride $(0.5 \% ; 2.5 \%)=>$ for salt taste One drops of each tastant was presented to the four regions of the tongue in two concentrations using plastic disposable sterile pipette. The patients were asked to verify the taste as identified on a list presented to them. Results: Bitter taste was the most affected followed by salt then sweet and sour less affected on using low concentration while on using high concentration, Bitter taste was the most affected followed by the sweet then the salt and the sour is less affected. Anterior and posterior parts of tongue was equaly affected, with more affection of LT side posteriorly but no side preference anteriorly. Improvement was more rapid and obvious in anterior part of tongue than posterior part. Conclusion: Taste dysfunction may follow tonsillectomy operation (this appeared in $29 \%$ of cases of this study) and it's transient. Affection is slightly more on posterior part of tongue than anterior part. The most affected taste was bitter then sweet, salt and sour respectively.
\end{abstract}

Keywords: Dysgeusia, Tonsillectomy, Chemogustometry

\section{Introduction}

It is well known that tonsillectomy is the most commonly performed operation in the otolaryngologicalfield ${ }^{(1)}$ Although tonsillectomy is regarded as a safe surgery, it still has the risk of per- and postoperative complications such as anaesthetic complications, primary or secondery haemorrhage, severe nausea, dehydration, infection, velopharyngeal insufficiency and nasopharyngeal stenosis ${ }^{(2)}$. However, taste disturbance is an unusual complication with few reports on it ${ }^{(3)}$.

There are several possible causes of taste disturbance: (I) injury of the lingual branch of the glossopharyngeal nerve (LBGN) when the inferior part of the palatine tonsil is removed, (II) pressure of the retractor on the tongue, (III) Zinc deficiency due to lack of intake after surgery and (IV) adverse effects of medications ${ }^{(4)}$.

Even it doesn't threaten directly the patient's life; it has a considerable impact on life quality ${ }^{(5)}$. Altered taste perception seems to be more frequent than previously thought. Fortunately, most of prospective studies show that altered taste perception remains a transient phenomenon with total recovery after several weeks ${ }^{(6)}$.However, long-lasting 
and disabling dysgeusia, such as absent, but more often badly distorted taste perception has repeatedly been described after tonsillectomy ${ }^{(5)}$.

The chemical senses and their normal functioning constitute a necessity for a good quality of life as the gustatory and olfactory systems influence to a large extent food selection and affect nutrition (7) Dysfunction of gustatory system can have detrimental effects on a patient's health ${ }^{(8)}$, possibly leading to depression in rare cases ${ }^{(9)}$.

Taste perception is mediated by individual taste buds, which located mainly in the primary taste organ; tongue ${ }^{(10) \text {. }}$

Unlike other sensory modalities like audition, vision or olfaction which relay on enervation of one nerve, the perception of taste is derived from the interaction of paired facial, glossopharyngeal and to lesser extent vagal nerve.

The component of facial nerve include the chorda tympani nerve and the greater superficial petrosal nerve (GSPN). The chorda tympani nerve receives taste informations from taste buds on the anterior $2 / 3$ of tongue, while the GSPN receives taste perception from the palate ${ }^{(11)}$.

The glossopharyngeal nerve receives taste sensation from taste buds on posterior $1 / 3$ of tongue mediated by lingual branch of glossopharyngeal nerve ${ }^{(11)}$.

The taste fibers in the epiglottis are innervated by the superior laryngeal branch of the vagus ${ }^{(10)}$.

The sensation of taste can be categorized into four basic tastes: sweetness, sourness, saltiness, bitterness.

Application of taste substances is the method of taste examination. There are various methods of applying taste substances during gustometry examination. The stimuli used in gustometry are: citric acid (sour taste), caffeine or quinine hydrochloride (bitter taste), sodium chloride (salty taste), glucose (sweet taste) ${ }^{(12)}$.

\section{Patients and Methods}

\subsection{Patients}

This study was carried on 100patients, attending ENT out patient clinic in Menoufia Faculty of medicine hospital planned to do bilateral tonsillectomy, they was at least 10 years of age.

All patients were subjected to the following:

Full history taking

Thorough ENT clinical examination

Important areas of the physical examination in patients to exclude pre existing gustatory impairment (pre operative):

Ears: examination for status of middle ear for inflammations or any previous surgery.

Nose: Anterior rhinoscopy with an otoscope to look for nasal mass.

Oral cavity: examination of the tongue including presence, gross size, number, distribution of papillae, examination of the status of the health of the mucosa, the presence of adequate saliva, evidence of scar or previous surgery, visualization of and palpation for any oral cavity masses.

Neck: Palpation of submandibular, and sublingual areas for lymph nodes.

Psychiatric: General impression.

The exclusion criteria were:

1. Patients with preexisting taste disorders like:

Ageusia (complete: complete loss of taste, partial: loss of sensitivity in single taste quality)

Hypogeusia (gustatory hyposensitivity in comparison to young healthy subjects)

Hypergeusia (gustatory hypersensitivity in comparison to young healthy subjects)

Parageusia (is an inadequate or "wrong" taste sensation elicited by a taste stimulus)

Phantogeusia (means that patients report taste sensations, often described as metallic or salty, in the absence of a stimulus)

(Excluded by testing one day pre operative)

2. Diseases affecting gustatory function:

Xerostomia, Nutritional deficiencies, Renal impairment, Chemotherapy and radiotherapy, Diabetis mellitus

3. Regular drug use (table 3 )

\subsection{Methods}

Tonsillectomies were done by experienced surgeon with transoral suture ligation for haemostasis.

All tonsillectomies was performed under general anesthesia using Davis retractor.

Chemogustometry:

This was done one day prior to operation to assure intact and efficient taste sensation. Then one, seven, fifteen and thirteen days respectively post operative as follow up in abnormal findings.

One hour prior to testing all patients were asked not to eat or drink anything except water, and also not to smoke.

Four tastants with two concentrations for each were used: quininsulfate or caffeine $(0.075 \% ; 0.2 \%)=>$ for bitter taste, glucose $(2 \% ; 10 \%)=>$ for sweet taste ,citric acid $(0.5 \% ; 7.5 \%)$ $\Rightarrow$ for sour taste ,sodium chloride $(0.5 \% ; 2.5 \%)=>$ for salt taste.

One drops of each tastant was presented to the four regions of the tongue (anterior left, anterior right, posterior left, posterior right) in two concentrations (beginning with lowest concentration and ending with highest one) using plastic disposable sterile pipette. (32 trials for each patient $4 * 2 * 4=$ 4 tastant solutions $* 2$ concentrations for each solution*4 quadrants of tongue)

Mouth was rensed with purified water in between trials, presented to patient in plastic disposable cubs, discarded immediately after each patient.

An interval of thirty seconds was given between each trial.

Nostrils were occluded with vaslinated cotton to prevent odeur inhalation.

The patients were asked to verify the taste as identified on a list presented to them (sweet, salt, sour, bitter, water or no taste).

We used disposable plastic cubs and disposable plastic sterile pipette for every patient, and all disposed according the standards of infection control. 
Also solutions was prepared once weekly by adding weighted slats to measured amount of distilled water and preserved in fregirator.

\section{Results}

Age ranges from 10 to 49 years old, with mean age 17.41 and standard deviation of 9.42 (Table 1).

Table (1). Age difference of studied patients.

\begin{tabular}{lllll}
\hline & Minimum & Maximum & Mean & Std. Deviation \\
\hline Age & 10 & 49 & 17.41 & 9.42 \\
\hline
\end{tabular}

Time of operation ranges from 20 to 50 minutes, with mean time 33.27 and standard deviation of 8.99(Table 2).

Table (2). Time of operation for studied patient.

\begin{tabular}{lllll}
\hline & Minmum & Maximum & Mean & Std. Deviation \\
\hline $\begin{array}{l}\text { Time of } \\
\text { operation }\end{array}$ & 20 & 50 & 33.27 & 8.99 \\
\hline
\end{tabular}

Table (3). Sex difference of studied patient.

\begin{tabular}{lll}
\hline Sex & N & \% \\
\hline Male & 40 & 40 \\
Female & 60 & 60 \\
Total & 100 & 100 \\
\hline
\end{tabular}

Total number of studied patients (100) devided into 40 males and 60 females (Table 3).

Recognition percent for low concentration of four tastant solutions on both sides of tongue was as follow:

- Sweet tastant

On $1^{\text {st }}$ post operative day was $91 \%$ on RT and LT sides of tongue.

On $7^{\text {th }}$ postoperative day was $99 \%$ on RT and $98 \%$ on LT side of tongue.

On $15^{\text {th }}$ and $30^{\text {th }}$ postoperative day was $100 \%$ on RT and LT sides of tongue.

- Salt tastant

On $1^{\text {st }}$ postoperative day was $94 \%$ on RT and $95 \%$ on LT side of tongue.

On $7^{\text {th }}, 15^{\text {th }}$ and $30^{\text {th }}$ day postoperative days was $100 \%$ on RT and LT sides of tongue.

- Bitter tastant

On $1^{\text {st }}$ postoperative day was $86 \%$ on RT and $84 \%$ on LT side of tongue.

On $7^{\text {th }}$ postoperative days was $94 \%$ on RT and $93 \%$ on LT sides of tongue.

On $15^{\text {th }}$ and $30^{\text {th }}$ postoperative days was $100 \%$ on RT and LT sides of tongue.

- Sour tastant

On 1st postoperative day was $98 \%$ on RT and LT side of tongue.

On 7 th postoperative days was $99 \%$ on RT and $100 \%$ on LT sides of tongue.

On 15 th and 30 th postoperative days was $100 \%$ on RT and LT sides of tongue.
(Bitter taste was the most affected followed by salt then sweet and sour less affected) (Table 4).

Table (4). Recognition percent for low concentration of four tastant solutions on both sides of tongue.

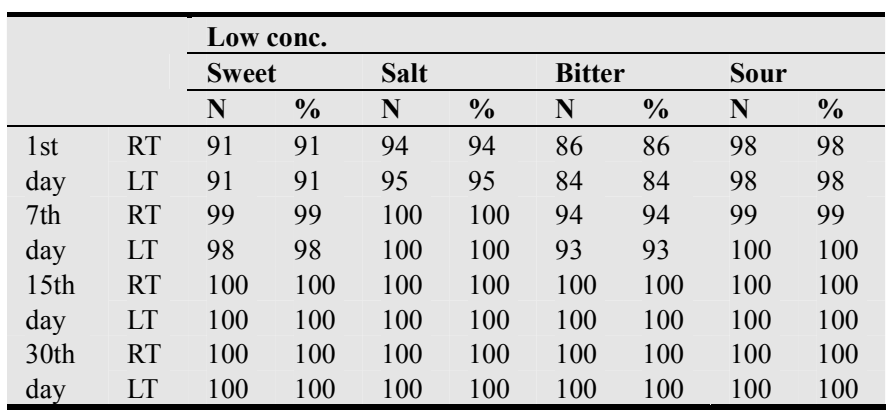

Recognition percent for high concentration of four tastants on both sides of tongue was as follow:

- Sweet tastant

On $1^{\text {st }}$ post operative day was $96 \%$ on RT and $95 \%$ on LT sides of tongue.

On $7^{\text {th }}, 15^{\text {th }}$ and $30^{\text {th }}$ postoperative day was $100 \%$ on RT and LT sides of tongue.

- Salt tastant

On $1^{\text {st }}$ postoperative day was $98 \%$ on RT and LT side of tongue.

On $7^{\text {th }}, 15^{\text {th }}$ and $30^{\text {th }}$ day postoperative days was $100 \%$ on RT and LT sides of tongue.

- Bitter tastant

On $1^{\text {st }}$ postoperative day was $87 \%$ on RT and $86 \%$ on LT side of tongue.

On $7^{\text {th }}$ postoperative days was $98 \%$ on RT and LT sides of tongue.

On $15^{\text {th }}$ and $30^{\text {th }}$ postoperative days was $100 \%$ on RT and LT sides of tongue.

- Sour tastant

On $1^{\text {st }}$ postoperative day was $99 \%$ on RT and LT side of tongue.

On $7^{\text {th }}, 15^{\text {th }}$ and $30^{\text {th }}$ postoperative days was $100 \%$ on RT and LT sides of tongue.

(Bitter taste was the most affected followed by the sweet then the salt and the sour is less affected) (Table 5).

Table (5). Recognition percent for high concentration of four tastant solutions on both sides of tongue.

\begin{tabular}{|c|c|c|c|c|c|c|c|c|c|}
\hline & & High & onc. & & & & & & \\
\hline & & Swe & & Salt & & Bitt & & Sou & \\
\hline & & $\mathbf{N}$ & $\%$ & $\mathbf{N}$ & $\%$ & $\mathbf{N}$ & $\%$ & $\mathbf{N}$ & $\%$ \\
\hline $1 \mathrm{st}$ & RT & 96 & 96 & 98 & 98 & 87 & 87 & 99 & 99 \\
\hline day & LT & 95 & 95 & 98 & 98 & 86 & 86 & 100 & 100 \\
\hline 7 th & RT & 100 & 100 & 100 & 100 & 98 & 98 & 100 & 100 \\
\hline day & LT & 100 & 100 & 100 & 100 & 98 & 98 & 100 & 100 \\
\hline 15 th & RT & 100 & 100 & 100 & 100 & 100 & 100 & 100 & 100 \\
\hline day & LT & 100 & 100 & 100 & 100 & 100 & 100 & 100 & 100 \\
\hline 30th & RT & 100 & 100 & 100 & 100 & 100 & 100 & 100 & 100 \\
\hline day & LT & 100 & 100 & 100 & 100 & 100 & 100 & 100 & 100 \\
\hline
\end{tabular}

Total number of patients who had gustatory dysfunction for one or more of four tastants presented to them in low concentration. 
(Comparison of impaired taste sensation for any of four tastants on anterior $1 / 2$ and posterior $1 / 2$ of tongue for both RT and LT sides).

- Anterior $1 / 2$ :

On $1^{\text {st }}$ day 16 cases was affected on RT side and 16 cases on LT side.

(15 cases with bilateral affection, 1 case with affection of right side only and 1 case affected only on left side)

On $7^{\text {th }}$ day 2 cases was affected on RT and LT sides.

On $15^{\text {th }}$ and $30^{\text {th }}$ day no cases of taste affection was detected.

- Posterior $1 / 2$ :

On $1^{\text {st }}$ day14 cases was affected on RT side and 16 cases on LT side.(13 cases show bilateral affection , 1 case affected only on right side and 3 cases affected only on left side)

On $7^{\text {th }}$ day 6 cases was affected on RT side and 6 cases on LT side.

On $15^{\text {th }}$ and $30^{\text {th }}$ day no cases of taste affection was detected.

(Anterior and posterior parts of the tongue was equal in total number of affected cases, with more affection of LT side posteriorly but no side preference anteriorly. Improvement was more rapid and obvious in anterior part of tongue than posterior part) (Table 6).

Table (6). Number of patients with gustatory dysfunction for any of four tastants after tonsillectomy operation.

\begin{tabular}{|c|c|c|c|c|c|c|c|c|c|}
\hline & & \multicolumn{2}{|c|}{$1^{\text {st }}$ day } & \multicolumn{2}{|c|}{$7^{\text {th }}$ day } & \multicolumn{2}{|c|}{$15^{\text {th }}$ day } & \multicolumn{2}{|c|}{$30^{\text {th }}$ day } \\
\hline & & $\mathbf{N}$ & $\%$ & $\mathbf{N}$ & $\%$ & $\mathbf{N}$ & $\%$ & $\mathbf{N}$ & $\%$ \\
\hline \multirow{2}{*}{ Ant. } & RT & 16 & 16 & 2 & 2 & 0 & 0 & 0 & 0 \\
\hline & LT & 16 & 16 & 2 & 2 & 0 & 0 & 0 & 0 \\
\hline \multirow{4}{*}{ Post. } & Total & 17 & 17 & 4 & 4 & 0 & 0 & 0 & 0 \\
\hline & RT & 14 & 14 & 6 & 6 & 0 & 0 & 0 & 0 \\
\hline & LT & 16 & 16 & 7 & 7 & 0 & 0 & 0 & 0 \\
\hline & Total & 17 & 17 & 10 & 10 & 0 & 0 & 0 & 0 \\
\hline
\end{tabular}

Taste affection on anterior part of tongue was mostly due to compression by mouth gag or prolonged time of operation rather than trauma of the nerve supplying this area (CTN), so improvement was more rapid and obvious on the few following days.

While on posterior part of tongue, taste affection was of slower improvement that inhances the hypothesis of gustatory dysfunction due to indirect trauma to the supplying nerve of this area (LBGN) during dissection of lower tonsillar part, without disruption of nerve fibers, so gustatory dysfunction was transient although improvement had slower rate than anterior part.

Number of incorrect recognitions for each of four tastants (some patients show affection of more than one tast).

Bitter taste was the most affected followed by sweet, salt and sour, with $17,10,6$ and 2 cases respectively on $1^{\text {st }}$ post operative day.

$7^{\text {th }}$ postoperative day showed regression in number of affected cases, bitter taste was most affected followed by sweet ,sour and salt taste, with 7, 2, 1, zero cases affected respectively.

$15^{\text {th }}$ and $30^{\text {th }}$ day shows no affected cases (Table 7).
Table (7). Total number of incorrect recognitions (wrong answers) for each tastant with low concentration.

\begin{tabular}{lllll}
\hline & $\mathbf{1}^{\text {st }}$ day & $\mathbf{7}^{\text {th }}$ day & $\mathbf{1 5}^{\text {th }}$ day & $\mathbf{3 0}^{\text {th }}$ day \\
\cline { 2 - 5 } & $\mathbf{N}$ & $\mathbf{N}$ & $\mathbf{N}$ & $\mathbf{N}$ \\
\hline Sweet & 10 & 2 & 0 & 0 \\
Salt & 6 & 0 & 0 & 0 \\
Bitter & 17 & 7 & 0 & 0 \\
Sour & 2 & 1 & 0 & 0 \\
\hline
\end{tabular}

\section{Discussion}

Tonsillectomy is among the most commonly performed operations, with the majority performed in the pediatric population $^{(13) .}$ It is generally regarded as a safe surgery, but otolaryngologists must be mindful of possible complications. The advancement in medical and surgical knowledge and technique has made this procedure safer, but the potential still exists for severe complications and long-term sequelae $^{(14)}$.

Complications of tonsillectomy can be further subdivided into intraoperative, immediate postoperative, delayed, and long term. Well-established delayed and longterm complications of tonsillectomy include hemorrhage, infection, dehydration, velopharyngeal insufficiency, nasopharyngeal stenosis ${ }^{(2)}$.

However, taste disturbance is an unusual complication with few reports on $i^{(3)}$.

There are several possible causes of taste disturbance: (I) injury of the lingual branch of the glossopharyngeal nerve (LBGN) when the inferior part of the palatine tonsil is removed, (II) pressure of the retractor on the tongue, (III) $\mathrm{Zn}$ deficiency due to lack of intake after surgery and (IV) adverse effects of medications ${ }^{(4)}$.

Taste distortions (including, dysgeusia and phantogeusia) after tonsillectomy are rarely reported as complications but have a significant impact on quality of life ${ }^{(2)}$. Fortunately, most of prospective studies show that altered taste perception remains a transient phenomenon with total recovery after several weeks ${ }^{(6)}$.

The chemical senses and their normal functioning constitute a necessity for a good quality of life as the gustatory and olfactory systems influence to a large extent food selection and affect nutrition (7) Dysfunction of gustatory system can have detrimental effects on a patient's health ${ }^{(8)}$; possibly leading to depression in rare cases ${ }^{(9)}$.

In comparison with the olfactory, auditory, and visual senses that depend on one cranial nerve $(\mathrm{CN})$, taste is mediated by three CNs: CN VII, CN IX, and CN X. The most well-studied and apparently important are the chorda tympani (CT) branch (innervating taste buds in the fungiform papillae on the anterior tongue) of CN VII and the lingual branch of $\mathrm{CN}$ IX (innervating taste buds in the foliate and circumvallate papillae at the posterior of the tongue). The role of CN X in taste perception is not well understood. ${ }^{(15)}$

This study was carried on 100patients, attending ENT out patientclilinic in Menoufia Faculty of medicine hospital planned to do bilateral tonsillectomy, they were at least 10 years of age. 
Aiming to asses taste function for the four basic tasts after tonsillectomy operation on anterior and posterior parts of tongue, devided into right and left sides.

The sensation of taste can be categorized into four basic tastes: sweetness, sourness, saltiness, bitterness.

Application of taste substances is the method of taste examination. There are various methods of applying taste substances during gustometry examination. The stimuli used in gustometry are: citric acid (sour taste), caffeine or quinine hydrochloride (bitter taste), sodium chloride (salty taste), glucose (sweet taste) ${ }^{(12)}$.

The present study presents data regarding incidence of gustatory affection after tonsillectomy operation in realation to age and sex of patient , time of operation , site of maximum affection on tongue(anterior or posterior, right or left ), and which of four tastants is more affected.

The data led to the following conclusions:

1. Gustatory dysfunction is not affected by age, sex of patient, or time of operation.

2. Gustatory dysfunction on posterior part of tongue is more obvious and persisting than anterior part.

3. Bitter taste is the most affected one of four basic tasts, followed by sweet then salt and lastly sour which is least affected.

In our study it was found that age (range from 10 to 49 years old, with mean age 17.41 and standard deviation of 9.42) and sex of the patients (40 males and 60 females) had no significant difference in post tonsillec to my dysguesia (PTD), which is in accordance with the results of Windfuhr. et al ${ }^{(16)}$ who had his study on 100 patients (40 male and 60 female) where they found no difference between both sex groups.

Regarding age of patients Stathas .et al ${ }^{(17)}$ who had his study on 60 patients with age range 14-40 years; mean 24.4 years; median 21 years; SD 7.7 yearsand found no significant difference in age.

In our study it was found that operation time (ranges from 20 to 50 minutes, with mean time 33.27 and standard deviation of 8.99) had no impact on gustatory dysfunction of patients.

The same was also observed in the study of Windfuhr .et al ${ }^{(16)}$ who had his study on 100 patients with operation time ranged from 15 to $60 \mathrm{~min}$ (mean 32.41, median 30, SD 13.3).

In our present study PTD was equal on anterior and posterior tongue within the first post operative day with $(17 \%)$ of patients failed to detect the stimulus anteriorly and posteriorly, while on seventh post operative day posterior tongue was more affected with failure percent $(10 \%)$ compared to anterior tongue (4\%), then improved on $15^{\text {th }}$ and $30^{\text {th }}$ day post operative with (0\%)failure of recognition.

While in the study done by Stathas. et al ${ }^{(17)}$ who found that posterior tongue was more affected than anterior tongue, test was done by applying 60 microliter of tastant solution on tongue. In the first post operative day $(47 \%)$ of patients failed to detect the stimulus anteriorly and (65\%) failed to detect it posteriorly, with improvement of recognition percent on subsequent follow up.

In our study the most affected taste was (bitter) taste with 17 affected patient, followed by (sweet) taste with 10 patients, then (salt) with 6 patients, and lastly (sour) taste with 2 patients on the $1^{\text {st }}$ post operative day. While on $7^{\text {th }}$ post operative day results was 7,2,0,1 respectively.

This comes in agreement with study of Stathas. et al (17) considering bitter taste the most affected one ,but not the same in considering sour taste the second most affected taste.

Bitter taste being the most affected and more persisting followed by sweet and salt supports the hypothesis of LGPN affection leading to decreased perception of bitter taste in posterior area of tongue supplied by this nerve more than anterior area of tongue supplied by CTN receiving sweet and salt. This comes in agreement with Goins and Pitovski ${ }^{(3)}$ that chorda tympani branch (innervating taste buds in the fungiform papillae on the anterior part of tongue) of CN VII and the lingual branch (innervating taste buds in the foliate and circumvallate papillae at the posterior part of the tongue) of CN IX.

This is supported by Windfuhr. et al ${ }^{(5)}$ that anterior tongue is particularly sensitive to sweet and salty stimuli, the posterolateral part to acidic taste and the posterior $1 / 3$ to bitter sensation. And also by Naik and Claussen ${ }^{(18)}$ That tip of the tongue is more sensitive to sweet. The sides of tongue are sensitive to salty in anterior part, whereas to sour in posterior part. Bitter is best tasted on the posterior third of tongue. The dorsal aspect of the tongue doesn't have many taste buds and is relatively insensitive.

Affection of LBGN was considered as indirect trauma during dissection or haemostas is of lower part of tonsils, this was confirmed by resolving of all cases with bitter taste disturbance within two weeks.

\section{Conclusion}

Taste dysfunction may follow tonsillectomy operation (this appeared in $29 \%$ of cases of this study), its transient. Age and sex of the patient is not relevant to the incidence.

Affection is slightly more on posterior part of tongue than anterior part thus inhances the possibility of being resulted from indirect trauma to lingual branch of glossopharyngeal nerve during dissection of lower tonsillar pole rather than compression by tongue elevator on anterior part of tongue during surgery.

The most affected taste was bitter then sweet, salt and sour respectively.

\section{References}

[1] Saito T, Honda N and Saito H: Advantage and disadvantage of KTP-532 laser tonsillectomy compared with conventional method. Auris Nasus Larynx 1999; 26:447-52.

[2] Vories AA: Dysgeusia associated with tonsillectomy. Head Neck Surg1999; 121:303-4.

[3] Goins MR and Pitovski DZ: Post tonsillectomy taste distortion: a significant complication. Laryngoscope2004; 114:120. 
[4] Tomita $\mathrm{H}$ and Ohtsuka K: Taste disturbance after tonsillectomy. ActaOtolaryngol2002; 546:164-72.

[5] Windfuhr JP, Schlo Endorff G, Sesterhenn A M, Kremer B: From the expert's office: localized neural lesions following tonsillectomy. Eur Arch Otorhinolaryngol2009; 266:1621.

[6] Tomofuji S, Sakagami M, KushidaK, Terada T, Mori H, Kakibuchi M: Taste disturbance after tonsillectomy and laryngo microsurgery. Auris Nasus Larynx2005; 32:381-6.

[7] Spielman AI: Chemosensory function and dysfunction. Crit Rev Oral Biol1998: 9:267-91.

[8] Deems DA, Doty RL, Settle RG, Gillon V M, Shaman P, Mester A F, Kimmelman C P, Brightman V J, Snow J B: Smell and taste disorders, a study of 750 patients from the University of Pennsylvania Smell and Taste Center. Arch Otolaryngol Head Neck Surg1991; 117:519-28.

[9] Scinska A, Jodkowska A, Korkosz A, Kukwa W, Sienkiewicz J H: Post-tonsillectomy dysgeusia with weight loss: possible involvement of soft palate. J LaryngolOtol2008; 122:5.

[10] Seiden A. Mand Brescia A. I.: The Anatomy and Physiology of Olfaction and Gustation, in Rhinology and Facial Plastic Surgery2009; 91-96.

[11] Kveton J. F. and Bartoshuk L. M.: The effect of unilateral chorda tympani damage on taste. Laryngoscope 1994; 104: 259.
[12] Klimacka-Nawrot E and Suchecka W: Methods of taste sensitivity examination. WiadLek2008; 61:207-10.

[13] Derkay CS.: Pediatric otolaryngology procedures in the United States: 1977-1987. Int J PediatrOtorhinolaryngol1993; 25:1-12.

[14] Colclasure JB and Graham SS.: Complications of outpatient tonsillectomy and adenoidectomy: a review of 3,340 cases. Ear Nose Throat J 1990; 69:155-160.

[15] Miller IJ. And Baroshuk LM.: Taste perception, taste bud distribution, and spatial relationships. In: Smell and Taste in Health and Disease (eds. Getchell TV, Doty RL, Bartoshuk LM, Snow JB). New York: Raven Press 1991; 205-233.

[16] Windfuhr JP, Sack F, Sesterhenn AM, Landis BN, Chen YS: Post-tonsillectomy taste disorders. Eur Arch Otorhinolaryngol2010; 267: 289-293.

[17] Stathas T, Mallis A, Naxakis S, Mastronikolis N S, Gkiogkis G, Xenoudakis D, Armenakis N S, Goumas PD.: Taste function evaluation after tonsillectomy: a prospective study of 60 patients. Eur Arch Otorhinolaryngol2010; 267: 1403-1407.

[18] Naik C. and Claussen C. F: Qualitative and quantitative representation of taste disturbances: How We Do It by Pentagon Chart. Indian J. Otolaryngol Head Neck Surg2010; 62(4):p.376-380. 